Reprod. Nutr. Dévelop., 1980, 20 (1 B), 295-299.

\title{
Récepteurs de l'hormone de croissance dans les membranes de foie de rat en insuffisance rénale
}

par Marie-Catherine POSTEL-VINAY, Joëlle FINIDORI, Christine KAYSER.

Unité de Recherches sur les Maladies du Métabolisme chez l'Enfant, U30

I.N.S.E.R.M., Hôpital des Enfants Malades,

149, rue de Sèvres, 75730 Paris Cedex 15.

Summary. Growth hormone receptors in liver membranes of rats with renal insufficiency.

Binding sites for human growth hormone (hGH) were studied in liver membranes of rats with chronic renal insufficiency (CRI) associated with a marked growth defect. A subtotal nephrectomy was performed in young female rats. One month after surgery, the rats with a plasma creatinine $\geqslant 3$ times that of controls were killed. The specific binding of 125/-hGH to microsomal membranes of rats with CRI was low. The number of binding sites rather than the binding affinity was affected; the number of both lactogenic and somatotropic sites was decreased. The sites of the plasma membranes as well as intracellular sites of Golgi fractions were reduced. No modification of the plasma GH and PRL was found. The hormone level did not appear to be the main regulating factor of the GH binding sites in this system.

\section{Introduction.}

Des sites de liaison pour l'hormone de croissance ont été identifiés dans le foie de rat (Kelly et al., 1974, Posner et al., 1975). Chez le rat femelle, deux types de sites sont présents : les uns à spécificité lactogénique, en plus grand nombre ; les autres à spéficité somatotropique (Kelly et al., 1974 ; Postel-Vinay, 1976).

Les rats en insuffisance rénale chronique (IRC) présentent un défaut de croissance, dont le mécanisme n'est pas clair. Le but de ce travail a été l'étude de l'interaction de l'hormone de croissance humaine $(\mathrm{hGH}$ ) avec les membranes de foie de rats en IRC. On sait qu'une altération de la réponse hormonale peut être liée à des changements de la liaison d'une hormone peptidique à ses récepteurs tissulaires.

\section{Matériel et méthodes.}

Animaux. - Des rats femelles (Charles River France, rats C.D.) pesant $100 \mathrm{~g}$ lors de l'intervention ont été séparés en 2 groupes. 
1) Un groupe de rats témoins a subi un simulacre d'intervention (laparotomie et ouverture de la capsule rénale).

2) Un groupe de rats a été néphrectomisé selon l'une des techniques suivantes : - néphrectomie des $7 / 8$ : totale d'un côté, bipolaire controlatérale ;

- néphrectomie totale unilatérale et électrocoagulation de la surface du cortex controlatéral ;

- néphrectomie des $7 / 8$ avec électrocoagulation du moignon restant.

Les animaux ont été nourris et abreuvés ad libitum. Le régime alimentaire a été soit celui fourni par I'UAR (Usine d'Alimentation Rationnelle) contenant 17,5 p. 100 de matières azotées, soit un régime fourni par I'INRA (Jouy-en-Josas) contenant 55 p. 100 de farine de poisson. Le poids et la taille (longueur tête-queve) des rats ont été mesurés chaque semaine sous anesthésie et le jour précédant le sacrifice. Les animaux ont été sacrifiés par décapitation sans anesthésie, un mois après intervention, entre $10 \mathrm{~h}$ et $11 \mathrm{~h}$ du matin. Les foies ont été prélevés et conservés à $4{ }^{\circ} \mathrm{C}$ pendant la préparation des membranes. Le sang de chaque animal a été recueilli dans des tubes héparinés pour le dosage des hormones peptidiques et de la créatininémie.

Préparation des membranes hépatiques. - Trois types de préparations ont été réalisés :

1) des fractions microsomales par centrifugation différentielle de l'homogénat de foie dans du sucrose $0,25 \mathrm{M}$;

2) des membranes plasmiques partiellement purifiées selon la méthode décriłe par Neville (1968);

3) des fractions de Golgi, selon la méthode d'Ehrenreich ; de l'éthanol est donné aux rats $90 \mathrm{~min}$ avant le sacrifice de façon à surcharger les éléments du Golgi en lipoprotéines de faible densité. Un gradient discontinu de saccharose permet de séparer des fractions de Golgi sur la base de leur densité.

Pour chaque préparation les foies des animaux néphrectomisés et des témoins ont été traités individuellement selon le même protocole ; les fractions préparées à partir des rats témoins d'une même série ont été regroupées en fin de préparation. Toutes les préparations membranaires ont été conservées dans l'azote liquide jusqu'à leur utilisation. Leur concentration en protéines a été estimée par la méthode de Lowry (1951) en utilisant l'albumine bovine comme standard.

Les membranes microsomales et plasmiques ont été caractérisées par l'activité de la $5^{\prime}$ nucléotidase, déterminée selon la méthode de Heppel (1955).

Mesure de la liaison des hormones iodées aux membranes hépatiques. - Les membranes $(0,1$ à $0,5 \mathrm{mg} / \mathrm{ml})$ ont été incubées avec l'hormone iodée à $20^{\circ} \mathrm{C}$ pendant $2 \mathrm{~h}$, dans $200 \mu l$ de phosphate $50 \mathrm{mM} \mathrm{pH} \mathrm{7,4,} \mathrm{avec} 1 \mathrm{mg} / \mathrm{ml}$ de sérum albumine bovine. A la fin de l'incubation l'hormone liée a été séparée de l'hormone libre par centrifugation. Des incubations ont été faites en présence d'un excès d'hormone native $(10 \mu \mathrm{g} / \mathrm{ml})$ et la liaison spécifique a été calculée comme la différence entre la quantité totale de radioactivité liée et celle qui n'est pas déplacée par un excès d'hormone native. 


\section{Résultats.}

Créatininémies. - Nous n'avons retenu pour notre travail que les rats ayant, lors du sacrifice, un taux plasmatique de créatinine supérieur ou égal à $15 \mathrm{mg} / \mathrm{l}$ c'est-à-dire 3 fois celui des animaux témoins (tabl. 1).

\section{TABLEAU 1}

Effets de la néphrectomie subtotale sur les taux plasmatiques de créatinine, d'hormone de croissance et de prolactine

\begin{tabular}{|c|c|c|c|}
\hline & $\begin{array}{l}\text { Créatinine } \\
\mathrm{mg} / 100 \mathrm{ml}\end{array}$ & $\begin{array}{l}\text { Hormone de croissance } \\
\mathrm{ng} / \mathrm{ml}\end{array}$ & $\begin{array}{l}\text { Prolactine } \\
\mathrm{ng} / \mathrm{ml}\end{array}$ \\
\hline $\begin{array}{l}\text { Rats « néphrectomisés » } \ldots \ldots . . . . \ldots \\
\text { Témoins } \ldots \ldots \ldots \ldots \ldots \ldots\end{array}$ & $\begin{array}{c}2,32 \pm 0,03 \\
0,58 \pm 0,02 \\
P<0,01\end{array}$ & $\begin{array}{c}40 \pm 5 \\
29 \pm 5 \\
\text { NS }\end{array}$ & $\begin{array}{c}89 \pm 19 \\
60 \pm 16 \\
\text { NS }\end{array}$ \\
\hline
\end{tabular}

Chaque valeur est la moyenne $( \pm \sigma \mathrm{m})$ de dosages chez 10 rats.

NS = différence non significative.

Croissance des rats (tabl. 2). - Le gain en poids ef en taille entre le jour de l'intervention et celui du sacrifice est plus faible chez les animaux néphrectomisés que chez les témoins. Le gain moyen en taille des rats néphrectomisés est de $56 \pm 4$ p. 100 par rapport à celui des témoins. Il existe une bonne corrélation $(p<0,001)$ entre créatininémie et croissance en taille ef également entre créatininémie et croissance en poids.

\section{TABLEAU 2}

Effets de la néphrectomie subtotale sur la croissance staturo-pondérale des rats

\begin{tabular}{lcc}
\hline & $\begin{array}{c}\text { Gain statural } \\
(\mathrm{cm})\end{array}$ & $\begin{array}{c}\text { Gain pondéral } \\
(\mathrm{g})\end{array}$ \\
\hline Rats néphrectomisés $\ldots \ldots$ & $5,01 \pm 0,3$ & $\begin{array}{r}44,07 \pm 5,7 \\
107,5 \pm 3,5 \\
\text { Rats témoins } \ldots \ldots \ldots \ldots\end{array}$ \\
\hline
\end{tabular}

Chaque valeur est la moyenne $\pm \sigma \mathrm{m}$ de mesures sur 17 rałs témoins, sur 15 rats néphrectomisés.

Taux plasmatique des hormones. - Les rats en IRC ef les rats contrôles ont des taux plasmatiques d'hormone de croissance et de prolactine qui ne sont pas significativement différents (tabl. 1).

Liaison de l'hGH, de la bGH et de la prolactine aux membranes hépatiques. - Une diminution de la liaison des 3 hormones a été trouvée dans les membranes microsomales des rais en IRC. L'activité moyenne de liaison aux membranes microsomales est de 40 p. 100 de celle des contrôles pour ${ }^{125}$-hGH, de 20 p. 100 pour ${ }^{125}$-bGH et de 50 p. 100 pour ${ }^{125}$-oPRL. Ces résultats indiquent que la diminution de la liaison porte 
sur les sites lactogéniques ef sur les sites somatotropiques. La concentration d'hormone native qui inhibe 50 p. 100 de la liaison de ${ }^{125}$-hGH est identique chez les animaux en IRC ef les témoins, ce qui suggère que c'est le nombre de sites plutôt que l'affinité de la liaison qui est diminué.

La diminution de la capacité de liaison pour l'hGH existe aussi dans les membranes plasmiques et dans les fractions de Golgi (fig. 1).

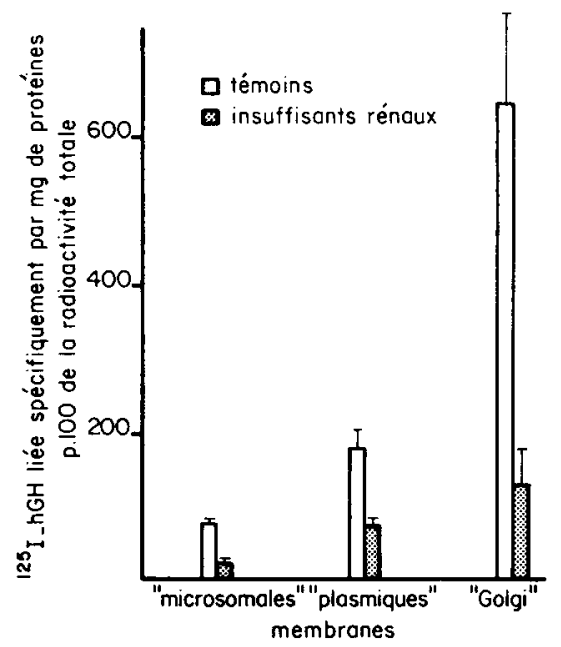

FIG. 1. - Liaison de ${ }^{125} /$-hGH aux membranes hépatiques de rats en insuffisance rénale. Les résultats sont exprimés par la moyenne ( $\pm \sigma \mathrm{m}$, indiquée par la petite barre verticale) d'expériences de liaison faite avec les membranes d'un nombre d'animaux allant de 3 à 15. La fraction membranaire indiquée « Golgi » sur l'abscisse est la fraction intermédiaire GF $_{2}$ du gradient discontinu de sucrose, selon la technique d'Ehrenreich.

\section{Discussion.}

Il existe une anomalie de la liaison de l'hormone de croissance aux membranes hépatiques de rats en IRC.

Parmi les rats ayant subi une néphrectomie subtotale, seuls (environ 20 p. 100 des animaux opérés) ont été étudiés ici, ceux dont l'insuffisance rénale, jugée sur le taux de créatinine, était sévère. Ces rats ont une croissance, en taille et en poids, très altérée. Les préparations de membranes hépatiques des rats en IRC et des témoins ne présentent pas de différence, si l'on en juge par leur activité en 5' nucléotidase. Il était intéressant de montrer l'existence d'un défaut de liaison de l'hGH aux membranes hépatiques de rats en IRC, de montrer que ce défaut est une diminution du nombre des sites de liaison, qu'il porte sur les sites lactogéniques et somatotropiques et qu'enfin, il concerne les sites localisés dans les membranes plasmiques aussi bien que les sites intracellulaires des fractions de Golgi.

Des exemples de relation entre le taux plasmatique d'une hormone peptidique et la concentration en récepteurs membranaires ont été établis récemment dans plusieurs modèles (revue de Tell et al., 1978). Il a été montré que la prolactine (Posner ef al., 1975) et l'hormone de croissance (Furuhashi ef al., 1978) sont capables d'induire 
leurs propres récepteurs dans le foie de rat. Dans notre système, les taux plasmatiques de prolactine ef d'hormone de croissance ne sont pas différents chez les rats en IRC et les témoins. Il apparaît donc que le défaut de liaison de l'hGH n'est pas sous le contrôle de la concentration plasmatique de l'hormone dans notre système. Quel que soit le mécanisme en cause, il ne touche pas tous les récepteurs des hormones peptidiques ; la liaison de l'insuline dans les membranes plasmiques est en effet plus élevée chez les animaux en IRC que chez les témoins. Ce résultat est à rapprocher d'une insulinémie basse chez les rats en IRC.

Dans notre modèle d'insuffisance rénale chronique où les rats présentent un défaut de croissance ef où le nombre des sites de liaison de l'hormone de croissance est diminué, la relation entre ces faiłs reste à examiner. Il est démontré que le foie est un organe de production de l'activité somatomédine et que cette production est sous le contrôle de l'hormone de croissance (Mc Conaghey, 1972). Il sera donc intéressant d'étudier les facteurs de croissance plasmatiques dans notre modèle. 5e Réunion du groupe Développement I.N.R.A.,
Clermont-Ferrand/Theix, 17-18 mai 1979.

Remerciements. - Les dosages radioimmunologiques de l'hormone de croissance et de l'insuline ont été faits par le Dr Rieutort ef le Dr Kervran, que nous remercions vivement. Nous sommes très reconnaissants envers le $\mathrm{Dr} C$. Kleinknecht qui a réalisé les néphrectomies des animaux.

\section{References}

EHRENREICH J. J. M., BERGERON P., SIEKEVITZ P., PALADE G. E., 1973. Golgi fractions prepared from rat liver homogenates. J. Cell Biol., 59, 45-72.

FURUHASHI N., FANG V. S., 1978. Hormone receptors in livers of $\mathrm{GH}_{3}$ tumor bearing rats : the predominant effect of growth hormone and testosterone. Endocrinology, 103, 2053-2060.

HEPPEL L. A., HILMOE B. J., 1955. 5' nucleotidase, p. 546. In COLOWICK S. P., KAPLAN W. O., Methods in Enzymology, vol. 11, Acad. Press, New York.

KELLY P. A., POSNER B. I., TSUSHIMA T., FRIESEN H. G., 1974. Studies on Insulin, growth hormone and prolactin binding : ontogenesis effects of sex and pregnancy. Endocrinology, 95, 532-539.

LOWRY O. H., ROSEBROUGH N. J., FARR A. L., RANDALL R. J., 1951. Protein measurement with the Folin phenol reagent. J. biol. Chem., 193, 265-275.

Mc CONAGHEY P., 1972. The production of suphation factor by rat liver. J. Endocr., 52, 1-9.

NEVILLE D. M., 1968. Isolation of an organ specific protein antigen from cell surface membrane of rat liver. Biochim. biophys. Acta, 154, 540-552.

POSNER B. I., KELLY P. A., FRIESEN H. G., 1975. Prolactin receptors in rat liver : possible induction by prolactin. Science, 188, 57-59.

POSTEL-VINAY M. C., 1976. Binding of human growth hormone to rat liver membranes : lactogenic and somatotropic sites. FEBS Letfers, 69, 137-140.

TELL G. P., HAOUR F., SAEZ J. M., 1978. Hormonal regulation of membrane receptors and cell responsiveness : a review. Metabolism, 27, 1566-1592. 